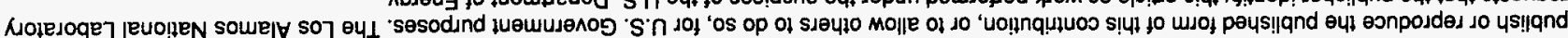

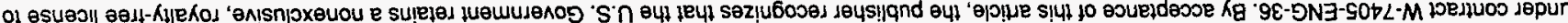

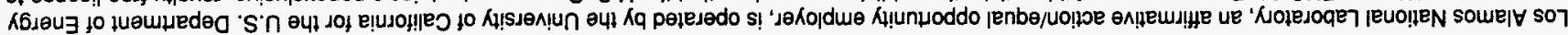

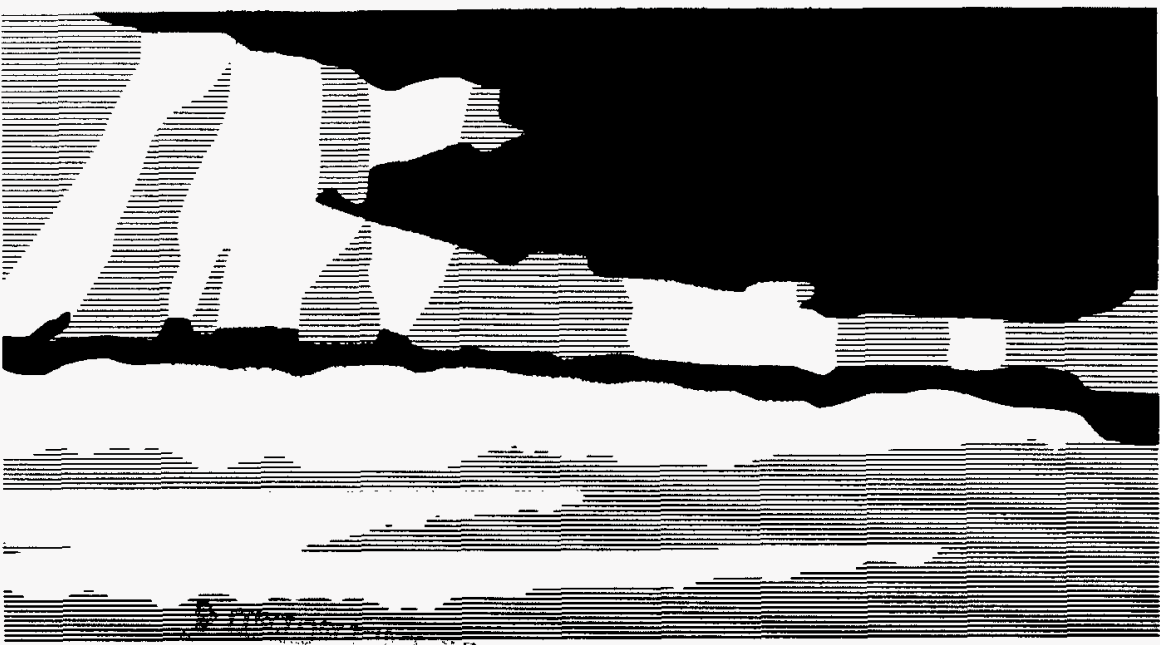

$\rightarrow T^{7}$

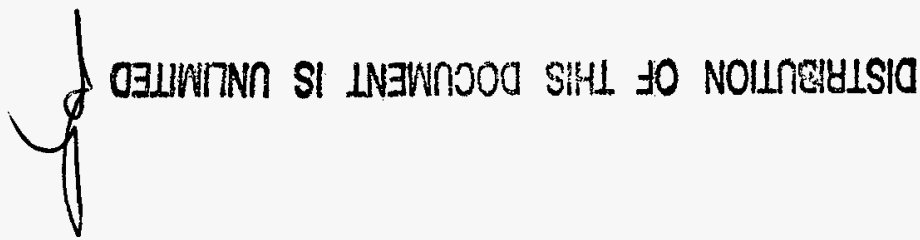

1.5

\section{0}

8SA: $90 \mathrm{MSH}$

0.15022U

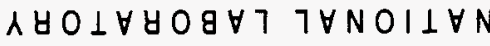 some|F So7}

191 EZt08661 uọnnq!גs!̣a jewaju|

:01 pan!uqua

:(s)JouInY

$\exists \perp \mathrm{COJ}$

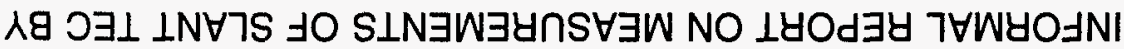

Kəssew 's :y

: 


\section{DISCLAIMER}

This report was prepared as an account of work sponsored by an agency of the United States Government. Neither the United States Government nor any agency thereof, nor any of their empioyees, makes any warranty, express or implied, or assumes any legal liability or responsibility for the accuracy, completeness, or usefulness of any information, apparatus, product, or process disclosed, or represents that its use would not infringe privately owned rights. Reference herein to any specific commercial product, process, or service by trade name, trademark, manufacturer, or otherwise does not necessarily constitute or imply its endorsement, recommendation, or favoring by the United States Government or any agency thereof. The views and opinions of authors expressed herein do not necessarily state or reflect those of the United States Government or any agency thereof. 


\title{
Informal report on measurements of slant TEC by FORTE
}

\author{
R. S. Massey
}

\section{Introduction.}

Los Alamos National Laboratory's Space and Atmospheric Sciences group is now operating the FORTE satellite, which has two sets of instruments: optical detectors and radio detectors. In this report I'll describe work with one set of radio deteciors that allow measurements of the total electron content (TEC) traversed by VHF radiation originating at an electromagnetic pulse (EMP) generator located at Los Alamos.

\section{Brief description of FORTE.}

The following description of the FORTE experiment is lifted directly from the FORTE web page at http://forte.lanl.gov. It has been edited to bring it up to date.

The FORTÉ payload consists of three instruments: an RF system, an optical system, and an "event classifier". The RF system incorporates three broad bandwidth RF receivers covering the frequency range 30-300 MHz, a polarization-selective antenna, and high speed waveform digitizers. The optical system consists of a coarse imager which is based on a NASA/MSFC design and has a $10 \mathrm{~km} \times 10 \mathrm{~km}$ ground resolution for lightning flash location $(500 \mathrm{frames} / \mathrm{sec})$ and a fast photodetector $(50 \mathrm{k}$ samples $/ \mathrm{sec})$ for recording individual light curves. The event classifier, based on digital signal processing technology, will provide on-orbit characterization of impulsive $R F$ events which have satisfied trigger criteria.

The complete FORTÉ project includes a small spacecraft and a satellite operations center at SNL in Albuquerque, New Mexico. The satellite will be nadir pointing and three axis stabilized. Body mounted solar cells will provide a daily averaged 55 Watts of power. The target orbit is $800 \mathrm{~km}$ altitude at $65^{\circ}$ inclination. Baseline designs specify a minimum mission duration of one year, with the capability to operate for up to three years. FORTE was launched aboard a Pegasus XI on August 29, 1997.

The RF system that was used to obtain data for this report is called the TATR, which is a $20 \mathrm{MHz}$ analogbandwidth receiver. The center frequency can be tuned from 30 to $300 \mathrm{MHz}$. The base-banded output is digitized at $50 \mathrm{MS} / \mathrm{s}$, with a 12-bit digitizer. The antenna used for these measurements is a log-periodic antenna with two arrays, to detect two linear polarizations. The antenna is about 10 meters long, and is nadir-pointing. The digitized signals are recorded in on-board solid-state memory, and downlinked to the control station at Sandia National Laboratory in Albuquerque. The digitizer runs continuously, and events are recorded when a multi-band triggering circuit determines that there has been a transient signal. Timing on the satellite (as well as location) is provided by an on-board GPS receiver.

For these experiments, the received signal came from an EMP generator called LAPP, for Los Alamos Portable Pulser. LAPP produces a ringing pulse of a few 10s of nanoseconds duration. with a center frequency (for these experiments) of about $45 \mathrm{MHz}$ and a useful bandwidth that extends beyond $150 \mathrm{MHz}$. 
Pulses are produced on a UT minute. with transmission time recorded by a GPS-based timing system to a few hundred ns (limited by GPS selective availability).

\section{Data and analysis techniques.}

In this section I'll show an example of the two ways we analyze the FORTE data to obtain the TEC. The data were taken with FORTE over $34.9^{\circ}$ north. $-103.2^{\circ} \mathrm{E}$, at an altitude of $821 \mathrm{~km}$. about $901 \mathrm{~km}$ from LAPP. Figure 1 is a perspective view of the earth as seen by FORTE. The elevation angle as viewed from Los Alamos was $67^{\circ}$. Figure 2 shows several waveforms from a LAPP shot. The receiver was configured to down-convert the $64-84 \mathrm{MHz}$ input to baseband. The top waveform in the figure is the base-banded waveform. The vertical scale is the electric field incident on the antenna, in volts/meter, and the horizontal scale is seconds. The chirped nature of the signal is immediately apparent. The middle waveform is the magnitude of the Hilbert transform, and the bottom plot shows the phase of the signal, in radians (also obtained from the Hilbert transform). A spectrogram of the waveform is show in figure 3. The frequency range plotted extends to the Nyquist frequency, but there is a $20 \mathrm{MHz}$ low-pass antialiasing filter ahead of the digitizer that causes the reduction in signals above $84 \mathrm{MHz}$. Again. the chirped nature of the signal is readily apparent. Some horizontal "gaps" in the spectrum are the result of a nondispersive filter that removes carriers from the television transmitters that use this band.

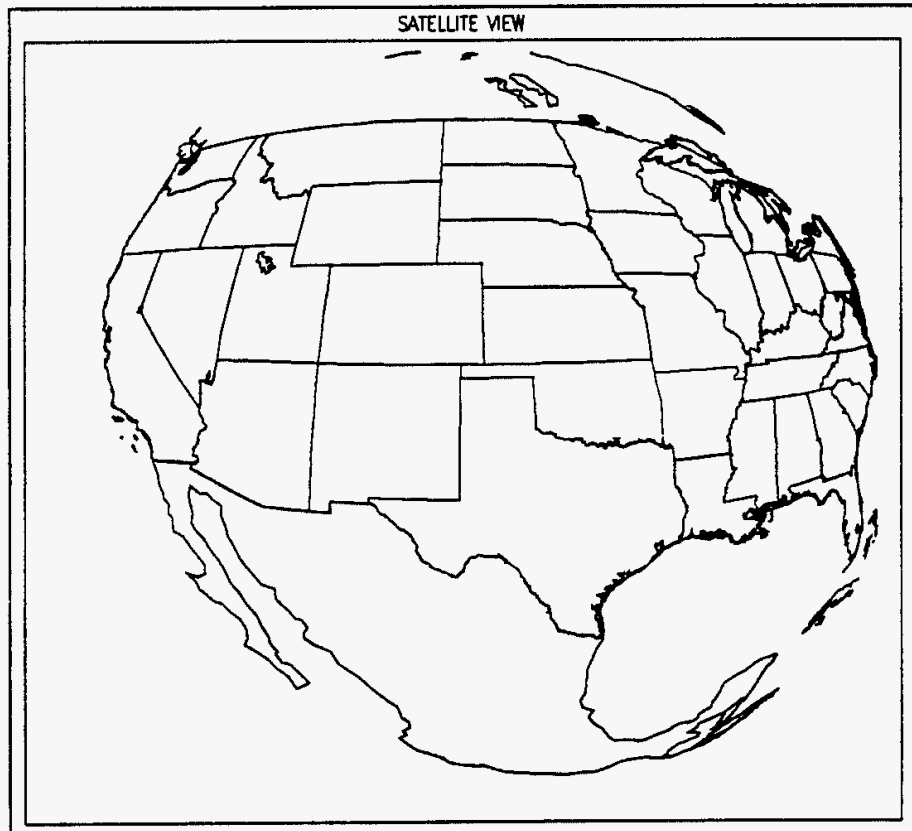

Figure 1. Perspective view from FORTE. 


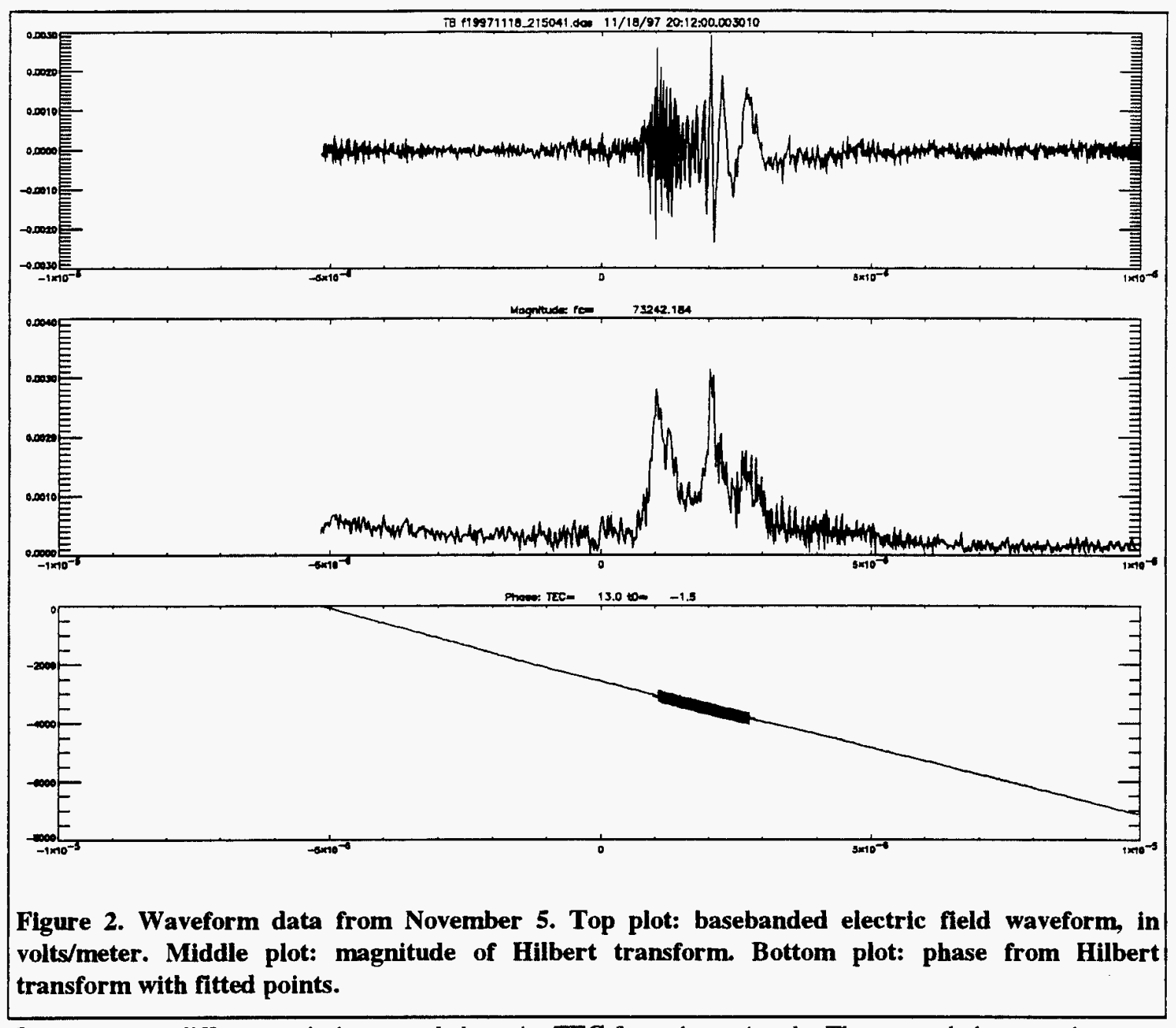

I use two very different techniques to deduce the TEC from these signals. The most obvious one is to use the spectrogram to obtain the group delay as a function of time, and to fit a curve of the form

$$
\tau_{\mathrm{i}}=\frac{\mathrm{kN}}{\mathrm{f}_{\mathrm{i}}{ }^{2}}+\mathrm{t}_{0}
$$

where $\tau_{\mathrm{i}}$ is the arrival time of the energy at frequency $\mathrm{f}_{\mathrm{i}}, \mathrm{k}=1.34 \times 10^{-7}$, and the TEC $\left(\mathrm{N}_{\mathrm{e}}\right)$ and $\mathrm{t}_{0}$ are unknowns. Figure 3 shows the results from this kind of analysis. The black dots on the spectrogram are the power-weighted centroid arrival times for that frequency bin, and the curve is the resulting fit, with the points weighted by the peak power at that frequency. The lowest frequencies in the band are not used, to minimize the effect of the higher-order terms in equation 1 , and the highest frequencies are avoided because of possible phase corruption by the anti-aliasing filter. The fitted vacuum time-of-arrival was -1.6 microseconds, and the TEC was 13.5 TEC units $\left(10^{16} \mathrm{~m}^{-3}\right)$. The formal uncertainty in the estimates is very small, but systematic errors are still being evaluated. 


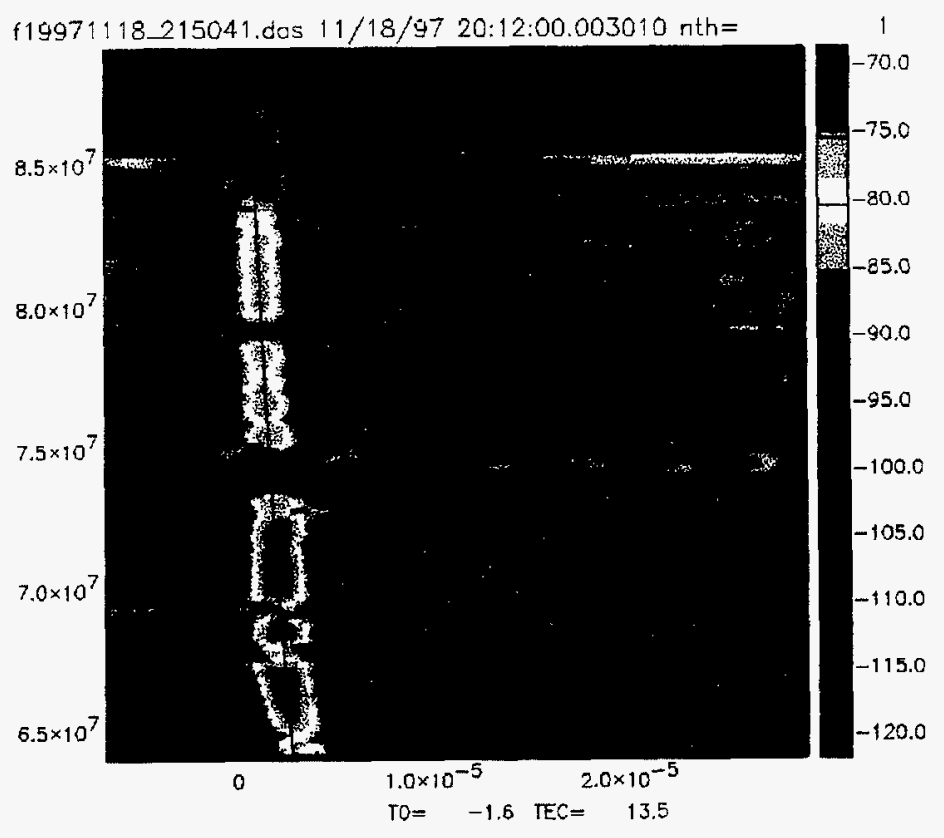

Figure 3. Spectrogram of the received pulse. The dispersion is measured as described in the text to obtain TEC and the corrected arrival time.

A numerically independent method of obtaining TEC is possible when the signal-to-noise ratio (SNR) is as high as it is here. We take the Hilbert transform of the signal to obtain the phase as a function of time, and fit those data to the relationship obtained by solving equation 1 for $f$, and integrating to obtain the phase:

$$
\phi=4 \pi \sqrt{\mathrm{kN}\left(\mathrm{t}-\mathrm{t}_{0}\right)}-\phi_{0}
$$

Now we have three parameters to solve for, which is undesirable, but the results at high SNR are quite impressive. The bottom plot in figure 2 shows the fitted phases for times at which the signal is strong enough for the phase measurement to be accurate. Figure 4 shows the difference between the measured phase and the fitted phase. The vertical axis is radians - the fit is very good. For this method, the deduced TEC was 13 TECU and $t_{0}$ was -1.5 microseconds. 


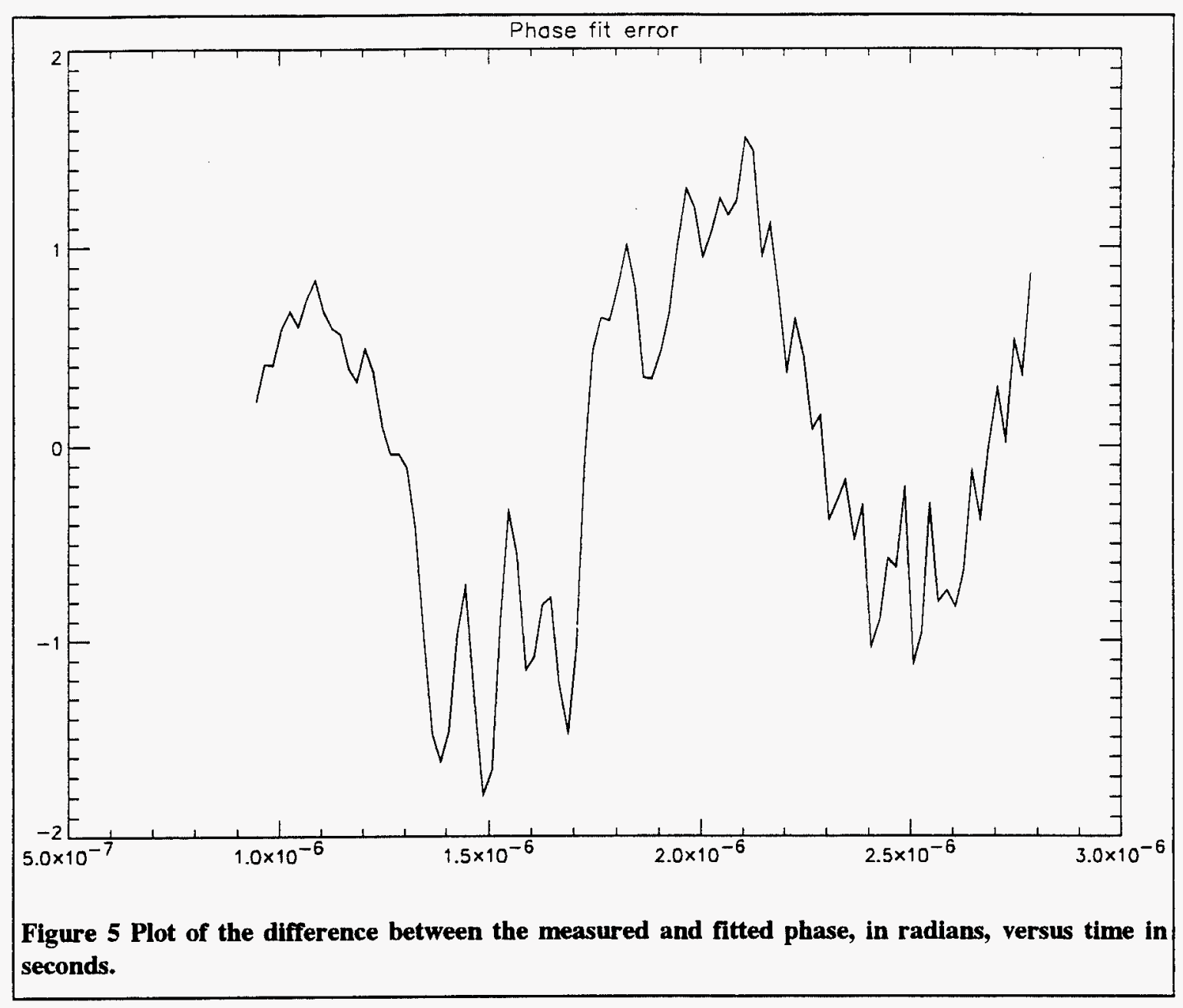

These two methods therefore arrive at essentially the same result.

\section{Results from three passes.}

We have obtained and analyzed good-quality data for three passes over Los Alamos. The first one was on November 5 (all dates and times will be UT unless otherwise noted). Both TATR receivers were used, and were tuned to receive $28-48 \mathrm{MHz}$, where dispersion is very pronounced. Table 1 summarizes the results from this run. 


\begin{tabular}{|r|r|r|r|r|r|}
\hline HH:MM & SS & AZ & EL & TEC A & TEC B \\
\hline $23: 22$ & 0.003 & 301.9 & 55.6 & 14 & 14 \\
\hline $23: 23$ & 0.003 & 248.6 & 67.3 & 13 & 15 \\
\hline $23: 24$ & 0.003 & 197.3 & 54.8 & 18 & 16 \\
\hline $23: 25$ & 0.004 & 181.2 & 38.6 & 24 & 23 \\
\hline $23: 26$ & 0.005 & 174.7 & 26.8 & 28 & 24 \\
\hline $23: 27$ & 0.006 & 171.3 & 18.5 & 41 & 67 \\
\hline $23: 28$ & 0.007 & 169.3 & 12.3 & 57 & 38 \\
\hline
\end{tabular}

Table 1. Results from November 5, 1997.
The first column gives the UT hour and minute of the event, and the second column gives the fractional seconds after the minute. $A Z$ and $E L$ are the azimuth (clockwise from north) and elevation above the horizon, in degrees, of the line of sight between the ground-based pulser and the satellite. The two slant TEC columns are the results from the $A$ and $B$ receivers, using the second analysis technique described above. The agreement between the two is quite good until the line of sight approaches the horizon, and the signal level drops to the point that the fits are becoming poor. We obtained from JPL the estimated vertical TEC to GPS, which was 15.5 directly over Los Alamos. I have not yet run an ionospheric propagation code to make the proper comparisons between these results.

The second pass occurred on November 13, with the results shown in table 2.

\begin{tabular}{|r|r|r|r|r|r|r|r|}
\hline UT & AZ & EL & RANGE & LAT & LON & ALT & TEC \\
\hline $22: 05: 00$ & 250 & 40 & 1188 & 32.9 & -114.5 & 819 & 26 \\
\hline $22: 06: 00$ & 225 & 36 & 1276 & 29.6 & -113.1 & 819 & 25 \\
\hline $22: 07: 00$ & 208 & 28 & 1478 & 26.4 & -111.8 & 820 & 32 \\
\hline $22: 08: 00$ & 197 & 21 & 1753 & 23.1 & -110.5 & 820 & 44 \\
\hline $22: 09: 00$ & 190 & 15 & 2073 & 19.8 & -109.4 & 821 & 45 \\
\hline $22: 10: 00$ & 186 & 10 & 2418 & 16.5 & -108.3 & 822 & 69 \\
\hline
\end{tabular}

Table 2. Results from November 13.

from the JPL data above Los Alamos was 16.6 TECU.

The final analyzed pass was on November 18 . The TATR A receiver was tuned to $120-140 \mathrm{MHz}$, and the TATR B receiver was tuned to 64-84 MHz. Cross-band data analysis (not yet done) can help to evaluate the effects of higher-order dispersion terms. Both types of analysis were done for TEC, but work on this run is incomplete. Table 3 shows the preliminary results. The vertical TEC above Los Alamos at the time was 16.5 TECU.

\begin{tabular}{|r|r|r|r|r|r|r|r|r|r|}
\hline UT & AZ & EL & RANGE & LAT & LON & ALT & CTEC @ 130 & CTEC @ 75 & ITEC @ 75 \\
\hline $20: 11: 00$ & 27 & 68 & 876 & 38.1 & 104.8 & 820 & & & \\
\hline $20: 12: 00$ & 107 & 67 & 901 & 34.9 & 103.2 & 821 & & 13.2 & 12.4 \\
\hline $20: 13: 00$ & 135 & 48 & 1102 & 31.6 & 101.7 & 821 & & 20.9 & 16.5 \\
\hline $20: 14: 00$ & 144 & 33 & 1397 & 28.3 & 100.3 & 821 & & 40.2 & \\
\hline $20: 15: 00$ & 147 & 23 & 1738 & 25.1 & 99.0 & 822 & 33.9 & 25.8 & \\
\hline $20: 16: 00$ & 150 & 16 & 2101 & 21.8 & 97.8 & 822 & 27.4 & 36.3 & \\
\hline $20: 17: 00$ & 151 & 10 & 2477 & 18.5 & 96.7 & 823 & 41.7 & & 49.2 \\
\hline
\end{tabular}

Table 3. Results from November 18.

In this table, CTEC refers to the TEC derived from the phase (the "coherent" technique). and ITEC refers to the "incoherent" group-delay technique. The data trom the $130 \mathrm{MHz}$ channel at high elevations has so little dispersion that I need to incorporate better receiver dispersion calibration before the results are meaningful.
For this run I've used the phase-analysis technique on data from the TATR A receiver, tuned to $28-48 \mathrm{MHz}$. There are several added columns in this table: the LATitude, LONgitude, range $(\mathrm{km})$ and ALTitude $(\mathrm{km})$ of the satellite at reception time. The fractional second column is omitted. The vertical TEC 


\section{Conclusions and plans.}

Analysis of data from the FORTE satellite can produce measurements of the slant TEC from a transmitter at Los Alamos to the satellite. These data should be useful in evaluating the TEC product that is derived by JPL from measurements of TEC by the GPS satellite constellation. We have planned for doing one of these passes per day, with some gaps, until we have covered a wide range of look angles and local times.

We will work with dual-band data to try to measure the effects of higher-order dispersion terms on the estimate of TEC. A GPS receiver at Los Alamos will be added to the JPL system, so that we'll have a better handle on the local GPS-derived TEC. We're also collecting ionogram data from Millstone Hill, which may be useful in constraining models.

We have a 3-D raytracing code, called TRACKER, that incorporates the ICED ionospheric mode. This code has options for doing transionospheric raytracing, and will be used to do detailed analysis of the FORTE results. 
M98004324

||||||||||||||||||||||||||||||||||||||||||||||||||||||||||||

Report Number (14) $L A-4 R-97-4852$

Publ. Date (11) $1997 / 1$
Sponsor Code (18) DOE/OP, XF
UC Category (19) UC-700, DOE/ER 